\title{
AUTOMATIC REGISTRATION, INTEGRATION AND ENHANCEMENT OF INDIA'S CHANDRAYAAN-1 IMAGES WITH NASA'S LRO MAPS
}

\author{
Vivek Kumar $^{1}$, Himanshu S. Mazumdar ${ }^{2}$ \\ ${ }^{1}$ Student, Information and Technology, Dharmsinh Desai University, Gujarat, India \\ ${ }^{2}$ Professor and Head, Research and development Center, Dharmsinh Desai University, Gujarat, India
}

\begin{abstract}
Chandrayaan-1 was India's first mission in deep space exploration to the moon. Its Terrain Mapping Camera (TMC) sent images of about 50\% of total lunar surface in its limited lifetime and covered polar areas almost completely at a high resolution of $5 \mathrm{~m} /$ pixel and 10m/pixel. This image dataset has been processed and put in public domain as individual strips of images categorized according to the orbits. The authors have already developed a Lunar GIS including a set of utilities like 3-D vision and exploration, crater detection and search using datasets from NASA's Lunar Reconnaissance Orbiter Wide Angle Camera (WAC) which are of lower resolution than CH1. The objective of this paper is to normalize and register the Chandrayaan-1 images to existing processed data so that all these utilities can be transparently applied to high resolution Chandrayaan-1 datasets. Registration process consists of identification of features in source and target images and estimating appropriate correction for offset, rotation and scaling parameters. Furthermore, due to the low altitude orbit of satellite, the acquired images have displacement of pixels from actual nadir position, which need non-linear correction. This paper describes step by step technique to integrate these high and low resolution images in single framework.
\end{abstract}

Keywords: Chandrayaan-1Lunar mapping, Moon, Feature based Image registration, Integration, ISRO, LRO, NASA, $T M C, W A C$.

\section{INTRODUCTION}

Lunar surface imaging is a major measurement in determining information about moon. It yields information about shape, surface and chemistry of moon. Recent years have seen a steady increase in lunar missions. These missions send huge amounts of imagery which is used in knowing more about the planets' surface and understanding structures like mare, ejecta, rings, craters and others. These datasets will be more useful if they can be integrated into single reference frame by aligning the images. Combining the datasets provides information about the topography and temporal changes, comparison, feature extraction, 3-D topography construction and mosaicing from stereo images. Image registration plays a crucial role to jointly exploit, integrate and compare these different datasets for enhanced information. The image registration process transforms a subject image so that it is geometrically aligned with a reference image. Most image registration methods can be broadly grouped into area based methods and feature based methods [1]. In area based methods, gray scale values from small window of pixels in the sensed image is compared statistically with window of the same size in the reference image. This is repeated over a search area in reference image and area of maximum match is found. These methods fail in unprocessed satellite images typically exhibiting lack of contrast and poor illumination [2]. Feature based methods extract low level features such as edges, curves, lines and corner points to estimate the alignment between images.
Registering images from different planetary missions is a challenging task. Varying orbital and temporal conditions of planetary missions often result in image coverage with widely varying resolutions and viewing geometries, gaps in coverage, poor image overlap, intensities, and have geometric variations like translation, rotation and projective [3].

The research group at DDU has developed a Lunar GIS using image datasets from LRO Wide Angle Camera. This dataset has a resolution of about $166 \mathrm{~m} /$ pixel. This system includes a set of utilities like $3 \mathrm{D}$ visualization and exploration of surface and crater detection. ISRO Data Center (ISSDC) has put higher resolution ( $5 \mathrm{~m} / \mathrm{pixel})$ image data of Chandrayaan1 Terrain Mapping Camera (TMC) in public domain. These images are contained in different strips which vary in height. The aim of this research is to register the ISRO's Chandrayaan-1 Terrain Mapping Camera (TMC) raw images with NASA's Lunar Reconnaissance Orbiter (LRO) global orthographic projection images.

The LRO dataset is in equi-rectangular projection and have been geometrically aligned. CH1 TMC images are raw orbital images. These images need coplanar correction with LRO for registration. In addition, $\mathrm{CH} 1$ had an orbit of 100 $\mathrm{km}$ for most of its duration. This low altitude orbit affects the across track imaging and pixels at far end need correction for topography. So image registration degrades as we go away from the center of the image towards the edges as discussed in Section 4.3. 
In this paper, a step by step registration method is described to automatically match the Chandrayaan 1 images with the existing LRO maps. The registered images will be integrated into the same application so that higher resolution $\mathrm{CH} 1$ images can be displayed seamlessly wherever possible and can be used in all developed utilities transparently.

This paper is organized as follow: The Section 2 contains summaries of some of the literature in planetary image registration. Section 3 describes about the Chandrayaan1 and LRO datasets and compares them. It also highlights the issues encountered during registration. In Section 4 the registration problem is formulated and various transformation needed to align images are discussed. Section 5 presents the formulated algorithm and Section 6 analyses the results of registration. Finally Section 7 concludes the paper with the summary of work.

\section{LITERATURE REVIEW}

Zitova and Flusser [1], describes the various approaches of image registration like area based and feature based method and classified them into subcategories according to the basic ideas of matching methods. Also the four basic steps of image registration procedure: feature detection, feature matching, mapping function design, and image transform with re-sampling are mentioned. This paper provides a comprehensive reference source in image registration, and surveys of the classical and up-to-date registration methods.

\subsection{Reviews of planetary feature detection}

Planetary surface contains complex shapes such as rocks, mountain, boulders, slopes and mostly craters. Craters were formed as a result of meteoroid impact and are of varying size and shape. Due to the relatively simple geometric shape of craters, they are easier to be detected by automatic image processing algorithms.

The literature on crater detections algorithms (CDAs) is extensive. Some studies make use of template matching [4] in global and local areas to detect craters. Some studies have attempted machine learning techniques like neural networks and support vector machine (SVM) [5, 6]. In machine learning algorithms, typically there are two phases, learning and detection. In the learning phase, the training set of images containing labeled craters is fed to an algorithm. In the detection phase, craters are detected in a new set of images using the trained algorithm. Wetzler et al. [7] tested a number of machine learning algorithms and reported that support vector machines achieve the best rate of crater detection.

Another study uses the combination of different algorithms to detect craters automatically in Mars images [8].

The Hough transform [9], an algorithm to detect line segments, and circular or elliptical shapes in images, plays an important part in several studies. A circle with radius $\mathrm{r}$ and center $\left(x_{c}, y_{c}\right)$ can be written as parametric equations:

$$
\begin{aligned}
& x=x_{c}+r \cos \theta \\
& y=y_{c}+r \sin \theta
\end{aligned}
$$

Hough transformation makes use of a voting process to find out the potential points which can be centers of the circle with enough pixels at the circular boundary. It iterates over all the edge pixels of the image and for all the possible values of $\theta$. When detecting circles of variable sizes, the Hough transform is then also repeated for all radii in the given interval. This makes the algorithm time consuming. Also in the images with low contrast, the circular features are not visible clearly. Therefore, edge detection does not give circles that would fit the simple circle model. In these cases, it is shown to have a lack of robustness in detecting the correct circles [10]. The algorithm accuracy is also limited in case of smaller craters where radii are about 2-5 pixels.

\subsection{Reviews of planetary feature matching}

A lesser number of studies are reported on matching of lunar optical images from different missions. Most existing researches make use of available laser altimeter data to coregister the images in the lunar and mars imagery.

Radhadevi et al. [11] presented a geometric correction method for Chandrayaan-1 imagery. The planimetric control was identified from the Clementine base map mosaic and vertical control was derived from LOLA data. However, this method only used Chandrayaan1 images from a limited area, and the control information was very limited.

Wu et al. [12] developed a bundle adjustment approach which was used match Chang'E-1 images with laser altimeter data for precise lunar topographic modeling. This model involves the laser altimeter points, EO parameters, and tie points which are collected from the stereo images.

Guo and $\mathrm{Wu}$ et al. [13] examined the co-registration between the DTMs derived from the Chang'E-1and SELENE laser altimeter data using a least squares surface matching method based on tie points identified from the DTMs.

Anderson et al. [14] examined the precision registration between Mars Orbiter Camera (MOC) imagery and Mars orbiter laser altimeter (MOLA) data at selected candidate landing sites. Kirk et al. [15] analyzed narrow-angle Mars Orbiter Camera (MOC-NA) images and produced Digital Terrain Models (DTM) to provide topographic and slope information. These DTMs are used in assessing the safety of candidate landing sites for the Mars Exploration Rovers (MER). These DTMs are controlled to the MOLA global data set and consistent with it at the limits of resolution of about $10 \mathrm{~m}$. Dickson et al. [16] registered MOC narrowangle images with interpolated MOLA data to analyze the distribution, local topographic setting, morphology, orientation, elevation, and slopes of Martian gullies in the $30-45^{\circ} \mathrm{S}$ latitude band.

Most of these methods ignore typical problems in planetary image matching, including the limited contrast, poor illumination, and the lack of distinct features.

Table - 1 summarizes the above studies.

where $\theta \in[0,2 \Pi]$. 
Table -1: Summary of studied planetary matching algorithms

\begin{tabular}{|c|c|c|c|}
\hline Authors & Title & Methodology & Results \\
\hline $\begin{array}{l}\text { J. Earl, A. F. } \\
\text { Chicarro, C. Koeberl, } \\
\text { P. G. Marchetti, and } \\
\text { M. Milnes }\end{array}$ & $\begin{array}{l}\text { Automatic Recognition of Crater-like } \\
\text { Structures in Terrestrial and Planetary } \\
\text { Images }\end{array}$ & $\begin{array}{l}\text { Hough transform and radial } \\
\text { consistency for automatic crater } \\
\text { detection }\end{array}$ & $\begin{array}{ll}\text { Automatic } & \text { Crater } \\
\text { recognition } & \end{array}$ \\
\hline $\begin{array}{l}\text { Bo Wua, Han Hua, } \\
\text { Jian Guo }\end{array}$ & $\begin{array}{l}\text { Integration of Chang'E-2 imagery and } \\
\text { LRO laser altimeter data with a combined } \\
\text { block adjustment for precision lunar } \\
\text { topographic modeling }\end{array}$ & $\begin{array}{l}\text { Bundle adjustment approach } \\
\text { using laser altimeter data, and } \\
\text { spacecraft parameters }\end{array}$ & $\begin{array}{lr}\text { Using } & \text { LRO laser data } \\
\text { with } & \text { Change-2 } \\
\text { imagery } & \end{array}$ \\
\hline $\begin{array}{l}\text { Bo Wu, Jian Guo, } \\
\text { Han Hua, Zhilin Li, } \\
\text { Yongqi Chen }\end{array}$ & $\begin{array}{l}\text { Co-registration of lunar topographic } \\
\text { models derived from Chang'E-1, } \\
\text { SELENE, and LRO laser altimeter data } \\
\text { based on a novel surface matching } \\
\text { method }\end{array}$ & $\begin{array}{l}\text { Surface matching technique to } \\
\text { detect the local deformation } \\
\text { using least-squares estimator }\end{array}$ & $\begin{array}{l}\text { Registration of } \\
\text { different lunar } \\
\text { topographic models }\end{array}$ \\
\hline $\begin{array}{l}\text { P.V.Radhadevi, } \\
\text { V. Nagasubramanian, } \\
\text { S.S.Solanki, } \\
\text { Krishna Sumanth T, } \\
\text { J. Saibaba, } \\
\text { GeetaVaradan }\end{array}$ & 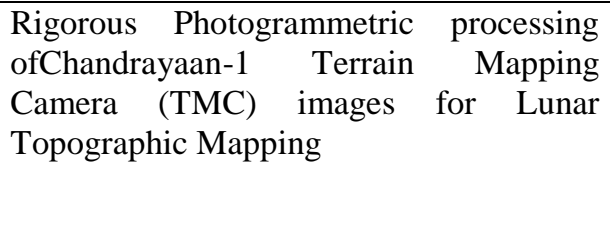 & $\begin{array}{l}\text { Geometric correction for } \\
\text { selected areas from TMC } \\
\text { imagery using LOLA vertical } \\
\text { control. }\end{array}$ & $\begin{array}{l}\text { Lunar topographic } \\
\text { images }\end{array}$ \\
\hline $\begin{array}{l}\text { Anderson, F.S., } \\
\text { Haldemann, A.F.C., } \\
\text { Bridges, N.T., } \\
\text { Golombek, M.P., } \\
\text { Parker, T.J., } \\
\text { Neumann, G }\end{array}$ & $\begin{array}{l}\text { Analysis of MOLA data for the Mars } \\
\text { exploration rover landing sites }\end{array}$ & $\begin{array}{l}\text { MOC images alignment to } \\
\text { MOLA profiles by matching } \\
\text { topographic features }\end{array}$ & $\begin{array}{l}\text { Precise topographic } \\
\text { registration for } \\
\text { potential landing sites }\end{array}$ \\
\hline $\begin{array}{l}\text { Kirk R., L., } \\
\text { Rosiek M. R., } \\
\text { Anderson J. A. }\end{array}$ & $\begin{array}{l}\text { Ultrahigh resolution topographic mapping } \\
\text { of Mars with MRO HiRISE stereo } \\
\text { images: Meter-scale slopes of candidate } \\
\text { Phoenix landing sites }\end{array}$ & $\begin{array}{l}\text { Rigorous geometric model } \\
\text { using EO parameters }\end{array}$ & $\begin{array}{l}\text { 3D maps at Phoenix } \\
\text { landing site }\end{array}$ \\
\hline $\begin{array}{l}\text { Dickson, J.L., } \\
\text { Head, J.W., } \\
\text { Kreslavsky, M. }\end{array}$ & $\begin{array}{l}\text { Martian gullies in the southern mid- } \\
\text { latitudes of Mars: evidence for climate- } \\
\text { controlled formation of young fluvial } \\
\text { features based upon local and global } \\
\text { topography }\end{array}$ & $\begin{array}{l}\text { Registration of MOC-NA with } \\
\text { interpolated MOLA dataset }\end{array}$ & $\begin{array}{l}\text { Analysis of } \\
\text { topography at Martian } \\
\text { gullies in the } 30-45^{\circ} \mathrm{S} \\
\text { latitude band }\end{array}$ \\
\hline
\end{tabular}

\section{DATASET}

DESCRIPTION

AND

\section{COMPARISON}

\subsection{Chandrayaan-1 Dataset}

Chandrayaan-1 (CH1) was an Indian Space Research Organization (ISRO) mission launched in October 2008[17]. It was designed to orbit the moon over a two-year period with the objectives of returning scientific information about the lunar surface. After one year, it suffered some technical difficulties and mission was declared as over. But in its limited lifetime, it managed to complete $95 \%$ of its objectives. The image dataset used is from the $\mathrm{CH} 1 \mathrm{TMC}$ and acquired from ISSDC
(http://www.issdc.gov.in/CHBrowse/index.jsp). The dataset is in Planetary Data System3 (PDS3) format. The dataset is in form of raw image data in .IMG files which contain 4000 (32 bit) floating point values per line forming the width of the image. The height of each image is different depending upon the imaging duration of orbiter at that time. The floating point values represent the pixel intensities which are then normalized to greyscale values according to camera and imaging characteristics. The $\mathrm{CH} 1$ images have been radiometrically corrected [18] but not geometrically aligned to match the lunar coordinates with actual position. Fig -1 shows the coverage of lunar surface coverage by the Chandrayaan1 TMC. 


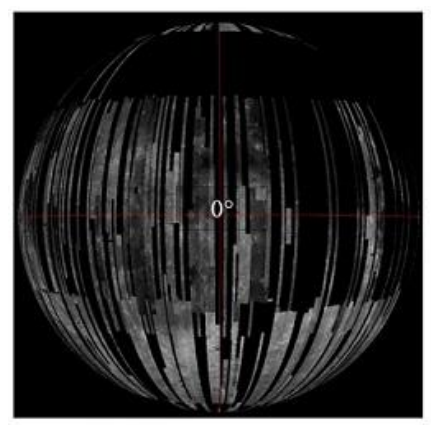

TMC Near Side Coverage

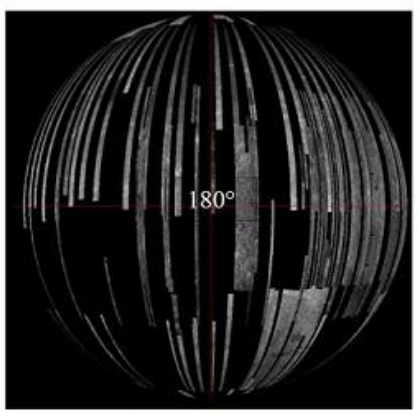

TMC Far Side Coverage

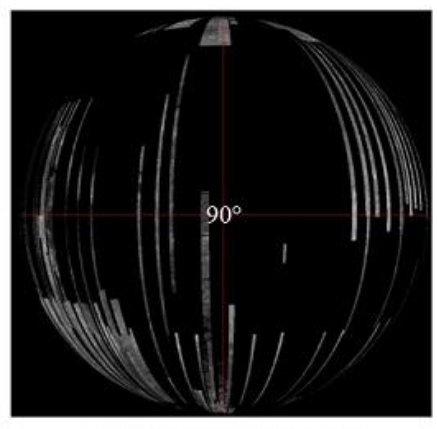

TMC $90^{\circ}$ EAST Coverage

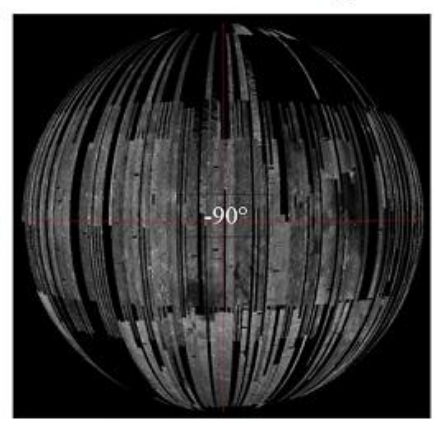

TMC $90^{\circ}$ WEST Coverage
Fig -1: Chandrayaan-1 TMC lunar surface coverage

Each image file is supplied with a detached label file and coordinates grid file. The label file (.LBL) contains general information about the image like imaging date and time, corner coordinates, image width and height, instrument names orientation. The supplied grid file (.TAB) contains the selenographic coordinates (Longitude, Latitude) of the pixels in image at an interval of 100 pixels. But these coordinates have some inaccuracies depending upon area, relief and errors in $\mathrm{CH} 1$. The coordinates of intermediate points can be interpolated assuming linear changes in coordinates at that level. This information is used for calculating the initial parameters of image registration and to decide the search space for matching the images.

\subsection{Lunar Reconnaissance Orbiter (LRO) Dataset}

NASA's Lunar Reconnaissance Orbiter (LRO) is an unmanned mission from NASA launched on June 18, 2009. After a year of exploring and sending images for complete lunar surface, its objectives were extended with a unique set of science objectives [19]. LRO sent images for full lunar topographic surface and still is functioning and sending important data.

The LRO Wide Angle Camera (WAC) dataset is acquired from LROC PDS3 data archive website, http://wms.lroc.asu.edu/lroc/view_rdr/WAC_GLOBAL. This dataset has been extensively processed for precise topography and accurate mapping of lunar coordinates.

Figures 2 and 3 compare the images of both the datasets for same locations in which the contrast and illumination differences can be seen. The detailed comparison of both datasets are presented in Table -2 .

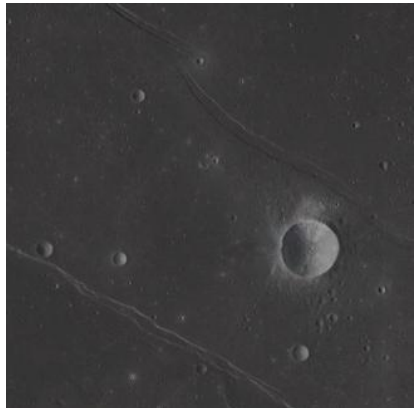

LRO WAC image

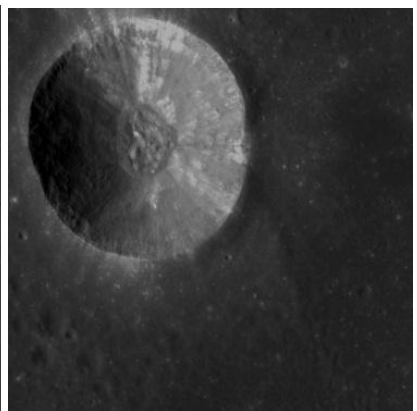

CH1 TMC Image
(Resolution $\sim 510 \mathrm{~m} /$ pixel) (Resolution $\sim 100 \mathrm{~m} /$ pixel)

Fig -2 LRO and $\mathrm{CH} 1$ images centered at about $38^{\circ} \mathrm{E}, 9^{\circ} \mathrm{N}$

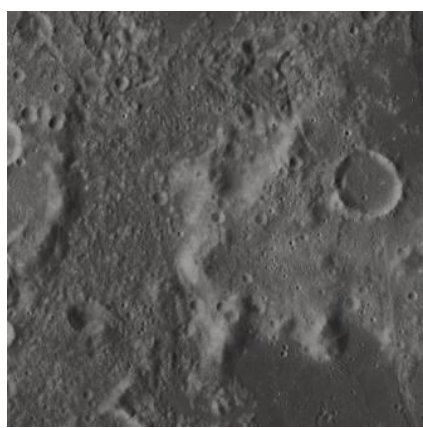

LRO WAC image

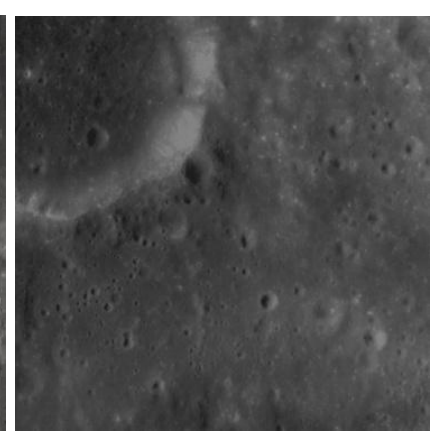

CH1 TMC Image
(Resolution $\sim 510 \mathrm{~m} /$ pixel) (Resolution $\sim 100 \mathrm{~m} /$ pixel)

Fig-3 LRO and $\mathrm{CH} 1$ images centered at about $41^{\circ} \mathrm{E},-4^{\circ} \mathrm{S}$

Table 2: Comparison of used datasets from LRO WAC and CH1 TMC

\begin{tabular}{|c|c|c|}
\hline Camera & $\begin{array}{l}\text { LRO Wide Angle } \\
\text { Camera (WAC) }\end{array}$ & $\begin{array}{ll}\text { CH1 } & \text { Terrain } \\
\text { Mapping } & \text { Camera } \\
\text { (TMC) } & \\
\end{array}$ \\
\hline Format & $\begin{array}{l}\text { Preprocessed jpg } \\
\text { images }\end{array}$ & $\begin{array}{l}\text { Raw PDS image, } \\
\text { No processing for } \\
\text { intensity, contrast, } \\
\text { or alignment }\end{array}$ \\
\hline $\begin{array}{l}\text { Image Size } \\
\text { (Pixels) }\end{array}$ & $512 \times 512$ & $\begin{array}{l}4000 \times \text { Variable } \\
\text { Height }\end{array}$ \\
\hline $\begin{array}{c}\text { Number of } \\
\text { images }\end{array}$ & $\begin{array}{ll}8192 \text { (64 rows } & \text { for } \\
\text { latitude changes and } \\
128 \text { columns } & \text { for } \\
\text { longitude) } & \end{array}$ & $\begin{array}{lr}1003 & \text { Strips of } \\
\text { different } & \text { heights } \\
\text { from } & \text { different } \\
\text { orbits } & \end{array}$ \\
\hline $\begin{array}{c}\text { Image } \\
\text { Resolution }\end{array}$ & $\sim 166 \mathrm{~m} /$ pixel & $\begin{array}{lll}5 \mathrm{~m} / \text { pixel } & @ & 100 \\
\mathrm{Km} & & \\
10 \mathrm{~m} / \text { pixel } & @ & 200 \\
\mathrm{Km} & & \end{array}$ \\
\hline $\begin{array}{l}\text { Image } \\
\text { Swath }\end{array}$ & $\sim 170 \mathrm{Km}$ & $\sim 20 \mathrm{Km}$ \\
\hline
\end{tabular}




\section{BACKGROUND THEORY}

\subsection{Registration Problem}

Image registration problem is to match and align an image, the sensed or floating image, onto another image, the reference image, by computing a transformation that is optimal in some sense [20]. The general problem consists of three elements:-

i. Reference image, IR $(x, y)$, that is kept unchanged

ii. Sensed image, IF $\left(x^{\prime}, y^{\prime}\right)$, that is transformed to match the reference

iii. Geometric transformation, $f$ that maps the two images

Two image locations in the reference and sensed image are said to be matched if they are mapped into each other under the geometric transformation:-

$$
I_{R}(x, y)=I_{F}\left(f_{x}\left(x^{\prime}, y^{\prime}\right), f_{y}\left(x^{\prime}, y^{\prime}\right)\right)
$$

Here, image registration problem is to devise an algorithm that, for all pairs of images, will compute the optimal transformations for any instance, where optimality depends on the choice of similarity measure S:-

$$
\alpha^{*}=\operatorname{ArgOptima}\left(S_{f}\left(I_{F}, I_{R}\right)\right)
$$

Here $\alpha^{*}$ represents the optimum value of transformation function $f$ with respect to similarity measure $S$.

\subsection{Transformations}

Transformation is estimated by using translation, rotation and scaling parameters. Mathematically, geometrical transformations that maps the points from one spatial coordinate $(x, y)$ to the other spatial coordinate $\left(x^{\prime}, y^{\prime}\right)$ can be written as:-

$$
\left(x^{\prime}, y^{\prime}\right)=T\{(x, y)\}
$$

where $\mathrm{T}$ is the transformation.

Affine transformation represents the relation between two images. It preserves points and straight lines but distances between points or angles between lines may change. Affine transformation is a combination of translation, rotation, along with scaling and or shear components. 2D affine transformation can be expressed as:-

$$
\left[\begin{array}{l}
x^{\prime} \\
y^{\prime}
\end{array}\right]=\left[\begin{array}{ll}
a 1 & a 2 \\
a 3 & a 4
\end{array}\right]\left[\begin{array}{l}
x \\
y
\end{array}\right]+\left[\begin{array}{l}
t x \\
t y
\end{array}\right]
$$

Here $a 1, a 2, a 3, a 4$ are affine transformation parameters and tx, ty are translation parameters.

\subsubsection{Translation}

Transformation can be two dimensional vectors or three dimensional vectors. It can be defined as how much amount of information or points or parallel shift of a figure moving from one position to the other with respect to the $x, y$ coordinate system.
Mathematically, the translation of point $P(x, y)$ by a vector $T$ is given by:-

$$
\begin{gathered}
\left(x^{\prime}, y^{\prime}\right)=(x+t x, y+t y) \\
{\left[\begin{array}{l}
x^{\prime} \\
y^{\prime}
\end{array}\right]=\left[\begin{array}{l}
x \\
y
\end{array}\right]+\left[\begin{array}{l}
t x \\
t y
\end{array}\right]}
\end{gathered}
$$

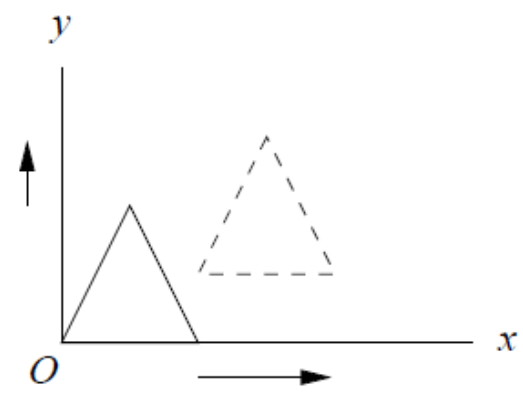

Fig -4 Translation

\subsubsection{Rotation}

Rotation is measured by degrees and defined as how much angle of the image is rotated. The new coordinates $\left(x^{\prime}, y^{\prime}\right)$ of a point $(x, y)$ rotated by $\theta$ (theta) radians around the origin, can be generated by the transformation:-

$$
\begin{aligned}
& x^{\prime}=x \cos \theta-y \sin \theta \\
& y^{\prime}=x \sin \theta+y \cos \theta \\
& {\left[\begin{array}{c}
x^{\prime} \\
y^{\prime}
\end{array}\right]=\left[\begin{array}{cc}
\cos \theta & -\sin \theta \\
\sin \theta & \cos \theta
\end{array}\right]\left[\begin{array}{l}
x \\
y
\end{array}\right] }
\end{aligned}
$$

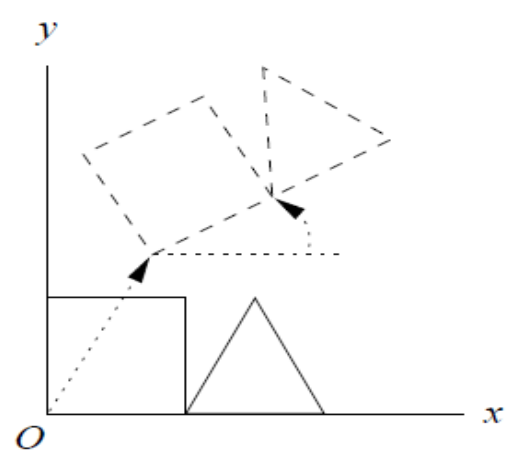

Fig -5 Rotation

\subsubsection{Scaling}

Rotation is measured by degrees and defined as how much angle of the image is rotated. The new coordinates $\left(x^{\prime}, y^{\prime}\right)$ of a point $(x, y)$ rotated by $\theta$ (theta) radians around the origin, can be generated by the transformation:-

$$
\begin{gathered}
x^{\prime}=s x^{*} x \\
y^{\prime}=s y^{*} y \\
{\left[\begin{array}{l}
x^{\prime} \\
y^{\prime}
\end{array}\right]=\left[\begin{array}{cc}
s x & 0 \\
0 & s y
\end{array}\right]\left[\begin{array}{l}
x \\
y
\end{array}\right]}
\end{gathered}
$$




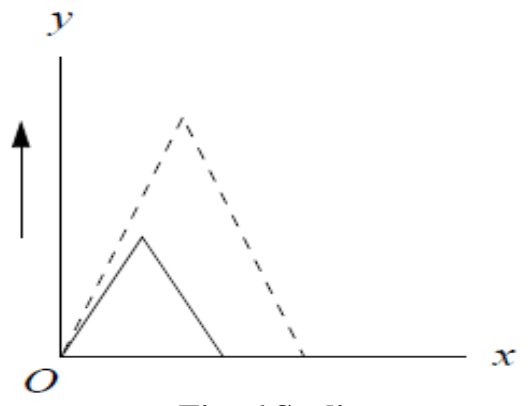

Fig -6 Scaling

\subsection{Non-linear Transformation due to low altitude}

Chandrayaan1 was in an orbit altitude of $100 \mathrm{~km}$ from lunar surface for most of its lifetime. Lunar surface has about 18 $\mathrm{km}$ range of variations from highest point to deepest point in the topography. At such low altitude imaging altitude, these variations produces error in image pixel displacement as shown in Fig -7. In the figure, A, B is actual position and A', $\mathrm{B}^{\prime}$ is position on datum plane. For features above the datum plane, the pixel on image are farther from nadir position and for features below datum plane, the perceived pixel on image are nearer to nadir which is shown by a", b" on the film plane.

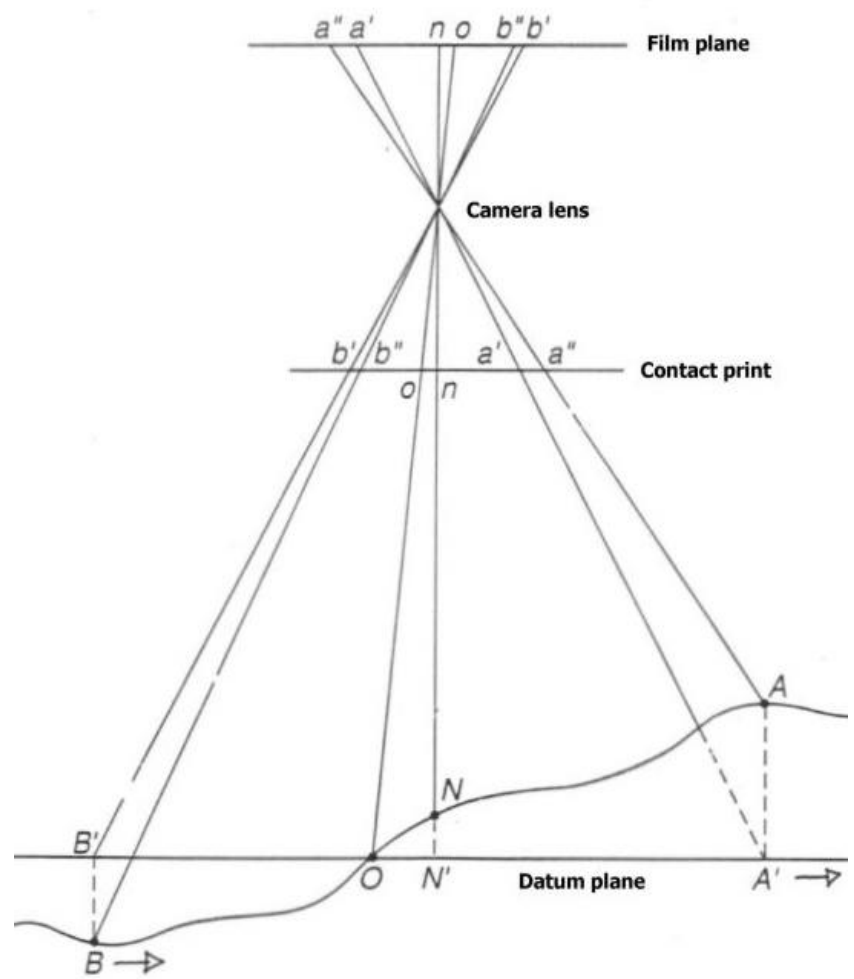

Fig -7 Schematic overview for terrain displacement due to surface topography variations. (Image source -

http://www.geog.ucsb.edu/ jeff/115a/lectures/geometry_of_ aerial_photographs_notes.html)

Non-linear corrections are required in each scan-line of $\mathrm{CH} 1$ TMC images for compensating these errors according to the height of surface at those points. These non linear corrections will warp the image edges.

\section{PROPOSED APPROACH}

The architecture of the proposed algorithm for registration of $\mathrm{CH}-1$ TMC images with the available LRO map database as shown in Fig -8, is as follows:-

Input: Images from both missions with coordinates $(x, y$, ) where $x$ is the Longitude, $y$ is Latitude. The coordinates for LRO WAC images are already available for each tile. Chandrayaan-1 TMC image coordinates will be calculated (interpolated) from the supporting grid files. each TMC file.

Desired Output: The corrected coordinates for

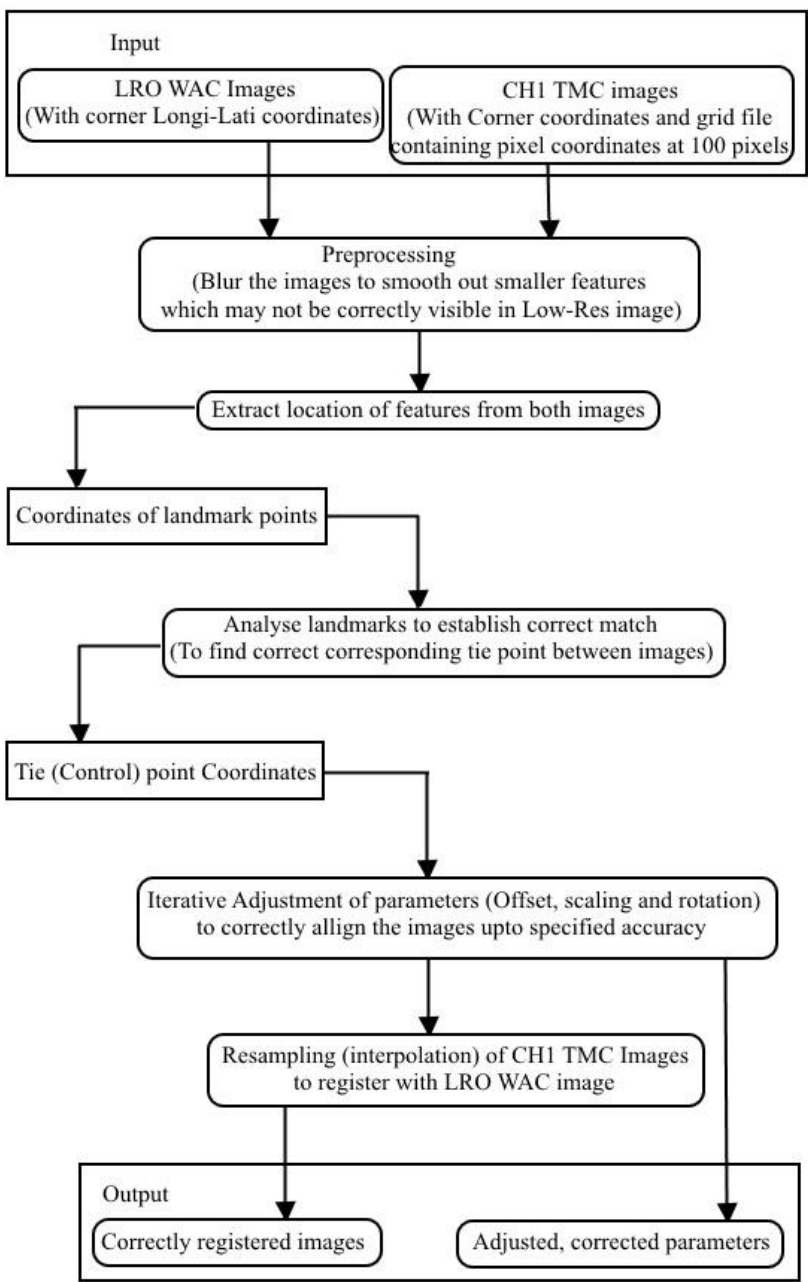

Fig -8: Flowchart for registration process

Steps:

i. Search the area according to the coordinates of given images. This will be approximate match. To establish the match, blur both images heavily to the same end level (more for TMC images and less for LRO images, as TMC has higher resolution). Blurring will ensure that only larger features get matched and smaller features which are present in TMC images and may result in mismatch, will be 
blurred out. Comparing the two images will give us a match factor.

ii. If match factor is more than a specified threshold, we will use these two images for further refining of the registration process.

iii. Improve the match using successive magnification, rotation and offset during random trials. This process will give us three correction factors, one for each of scaling, rotation, and offset.

iv. Select the image pair of low blurring or no-blurring and repeat the experiment with these factors, to further refine them.

[Note - This will still not account for non-linearity in images due to curvature and low altitude effects causing different magnification at different altitudes]

v. Recompose a new integrated image from corrected segments.

\section{EXPERIMENTS AND ANALYSIS}

The proposed method was examined using the LRO and TMC datasets. In the current experiments all TMC images are scaled down to the same resolution of the LRO images so that registration can be carried out. Since no previous research has attempted to register Chandrayaan-1 dataset with LRO before, we have compared the results of automatic registration process with the visually inspected coordinates of true registration.

Fig -9 shows the general setup for registration method. The left side image is from the LRO dataset and right one is from TMC dataset. The smaller dotted rectangle shows the placement of image as retrieved from using the supplied longitude-latitude coordinates. The solid black rectangle shows the search space for registration. Initially search space is kept about $30 \%$ in all directions from the supplied coordinates. The TMC image has been roughly resized to match the resolution of the LRO images. Once the correct location parameters have been found for the offset within the search space using the feature matching, the iterative process for refining the rotation and scaling parameters is done to enhance the registration process.

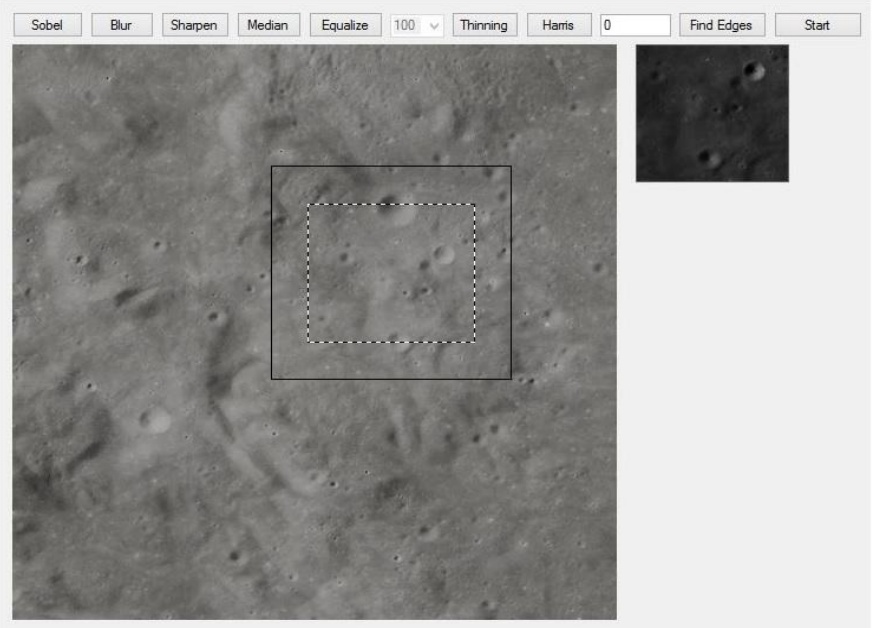

Fig -9: Experimental Search space for image registration
First, edge detection is done using Sobel Operator [21], which helps in detecting the prominent features. The edge map is added to the original image to enhance the edges in the image.

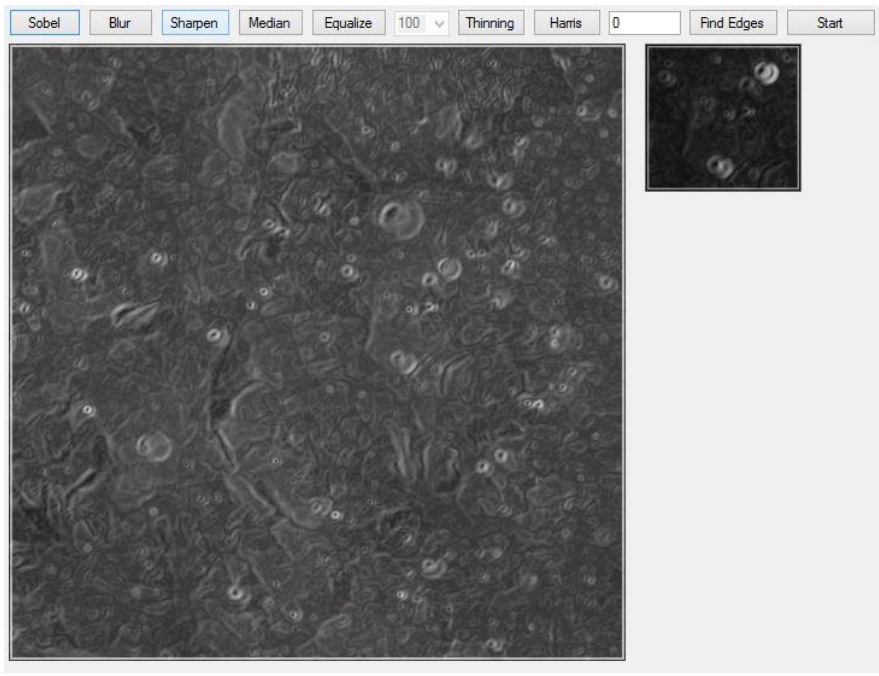

Fig -10: Sobel edge enhanced images

Then, features from both images are detected and matched as corners as shown in Fig -11.

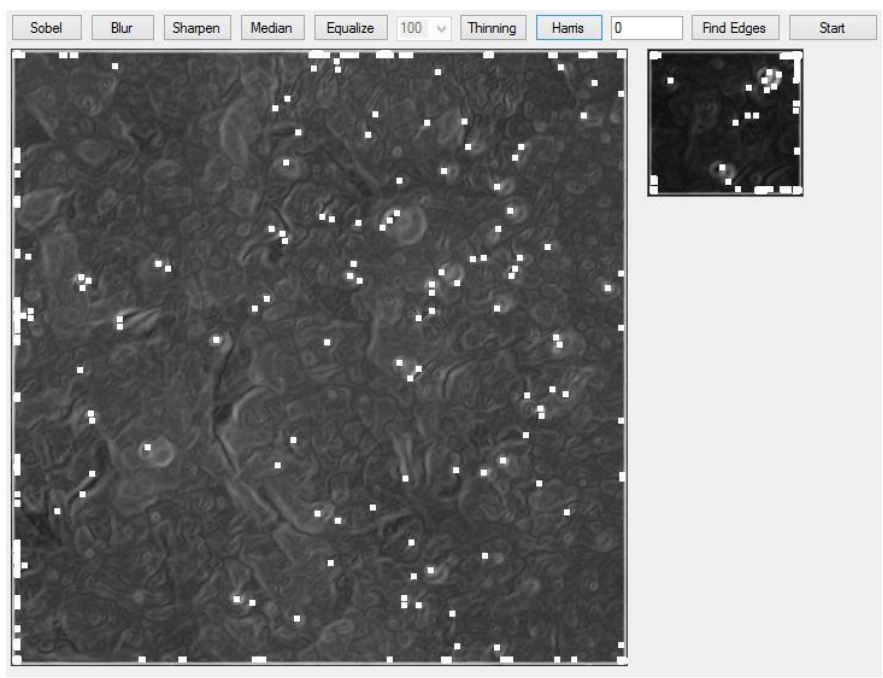

Fig -11: Detected corners in both images

Corners can be defined as points for which there are maxima or minima values for horizontal and vertical directions in the local neighborhood of the point. The TMC image is then moved over the search space to find out the position where the corners from both images are optimally matched.

After the initial matching, refining of the parameters is performed by iteratively changing the rotation and scaling parameters to increase the matching factor.

Below figures show some result images of registration found from the proposed method. The TMC images have been overlaid on the LRO images, according the parameters obtained from the approach. As can be seen from the results, the algorithm performs well even if the images have 
differences in the lightning directions and shadows but still have considerable features to match.

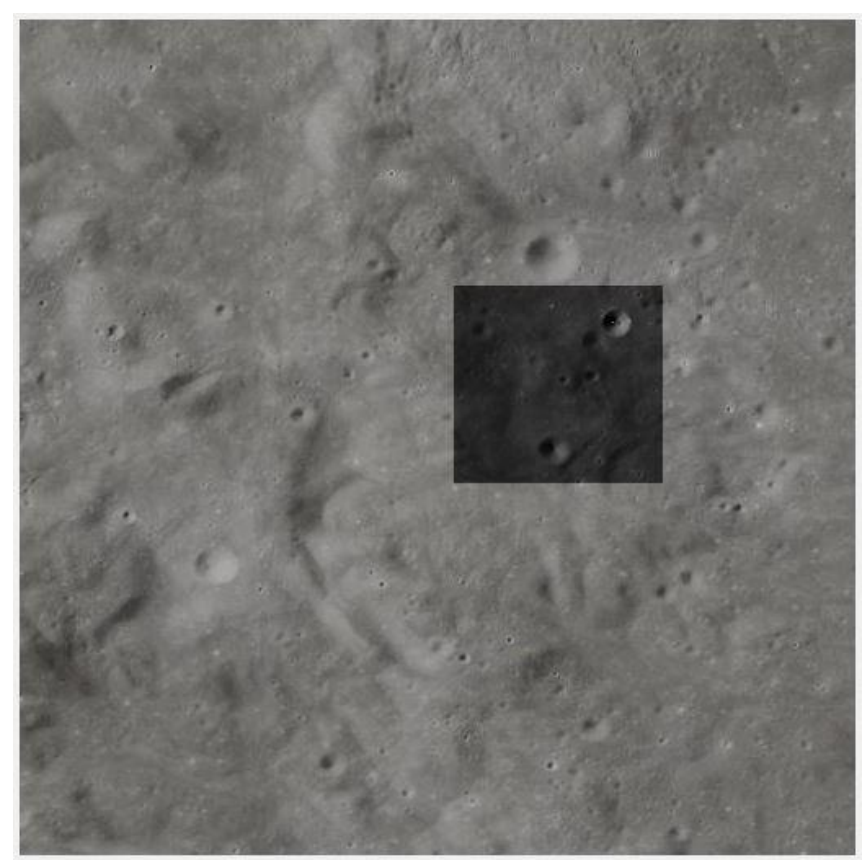

Fig -12: Registered image at $67.33 \mathrm{~W}, 28.56 \mathrm{~S}$

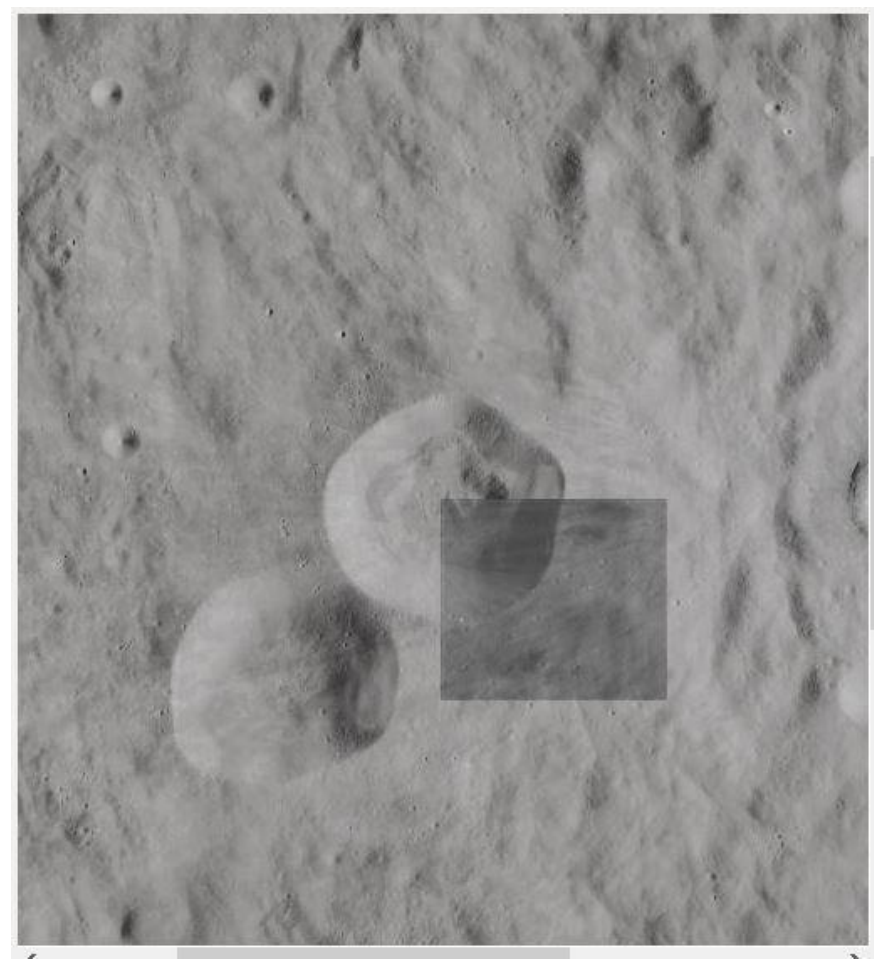

Fig -13: Registered image at 109.5W, 5.75N

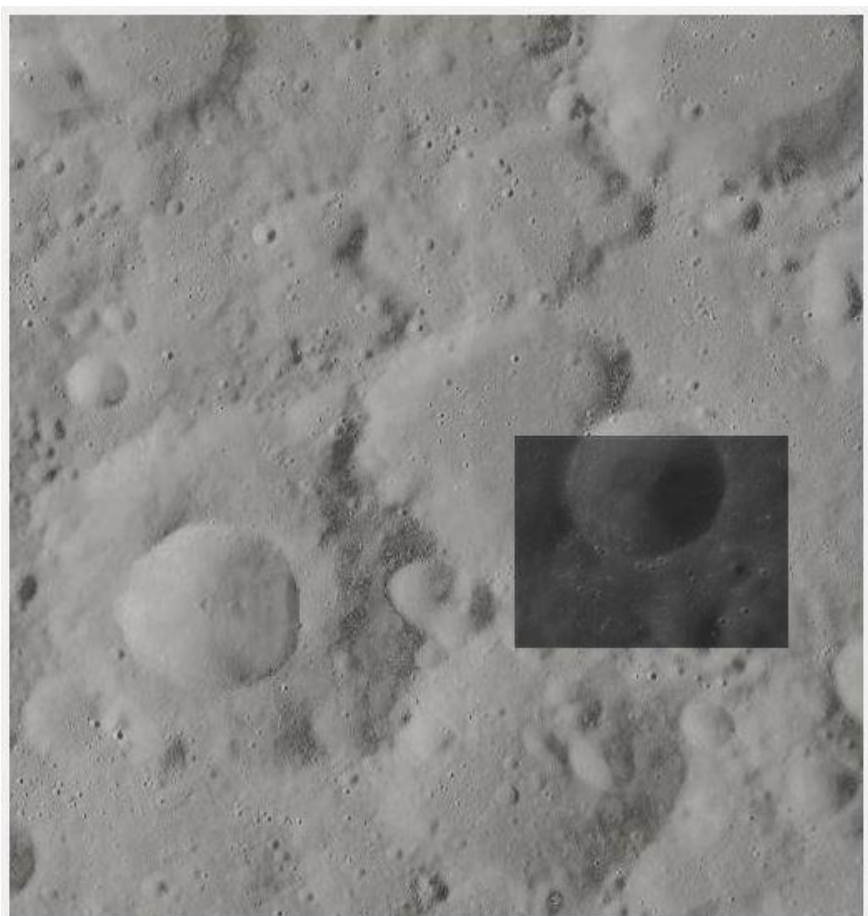

Fig -14: Registered image at 156.12W, 29.07N

Fig -14 and 15 show the areas where the method fails to find out acceptable results. This is due to failure to detect enough number of features to be matched.

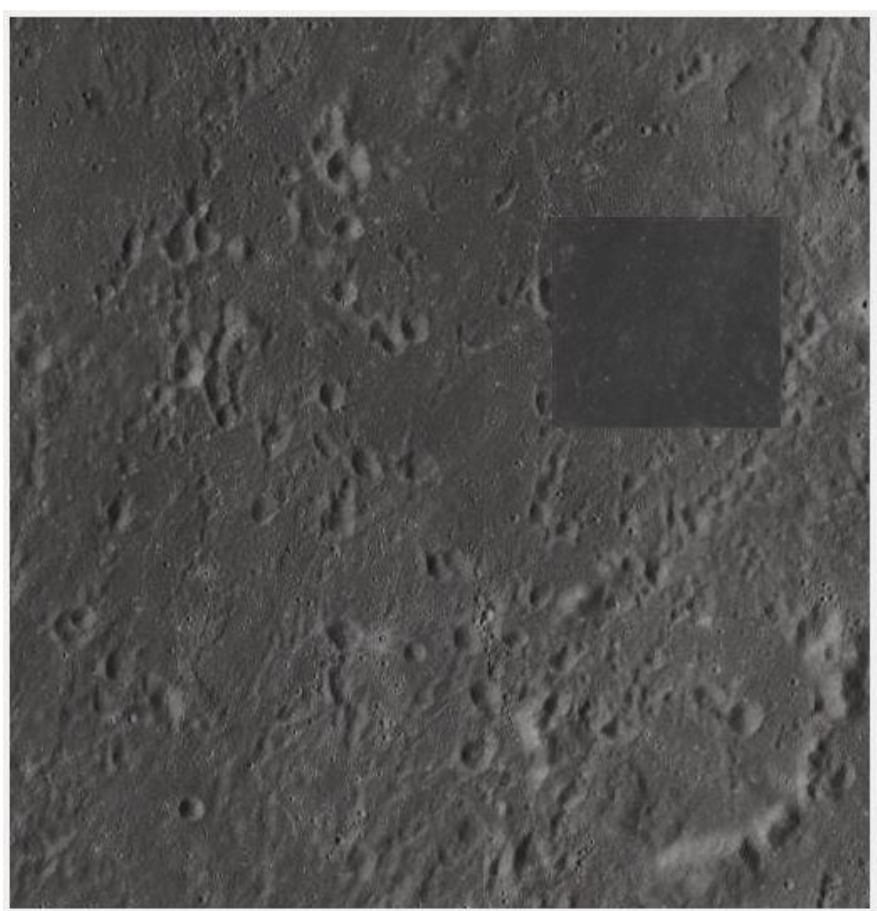

Fig -15: Wrong registration at 29.9E, 6.71S 


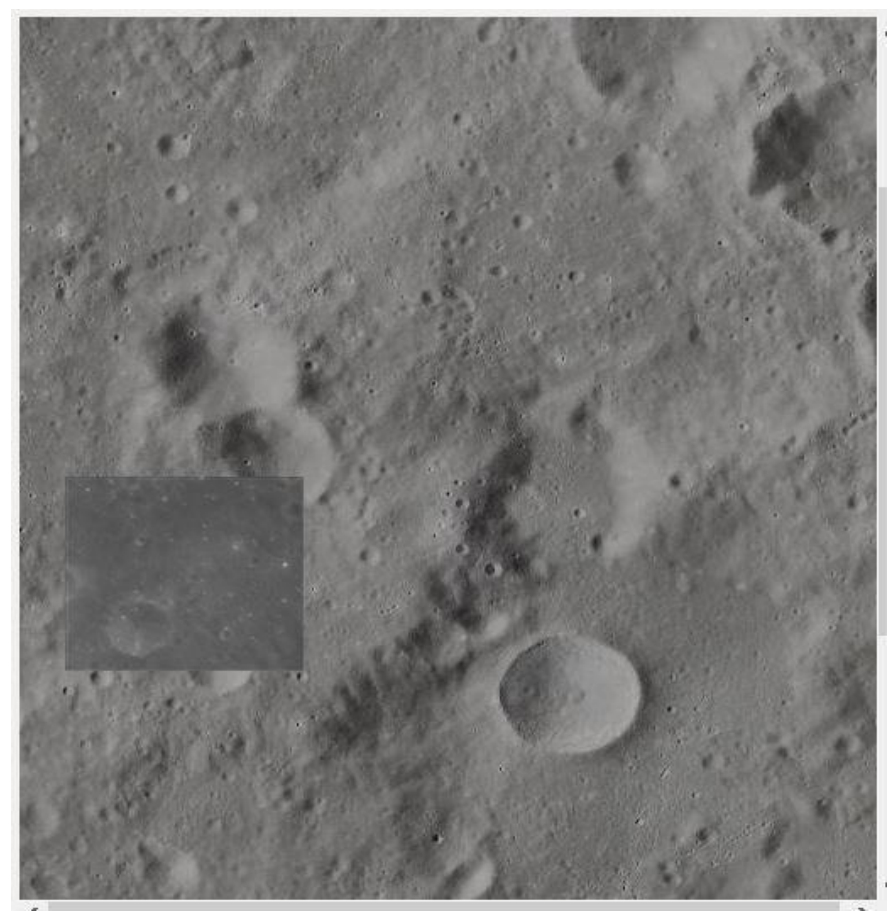

Fig -16: Wrong registration at 14.44E, 23.71S

\section{CONCLUSION}

An approach has been proposed in this paper for feature based extraction and matching of cross mission and cross sensor planetary images. These images are neither well contrasted nor have same coordinate systems which leads to changes in geometric projection of images.

This research focused on registration of lunar images from NASA's LRO mission and India's Chandrayaan1. Datasets from Chandrayaan1 TMC were analyzed and aligned with LRO WAC equi-rectangular projected maps. This method have still not considered the discrepancies in proper alignment due to topography of the moon. It can be refined to include the topography data from laser altimeter instruments to further increase the registration.

Experimental results demonstrated the feasibility and usefulness of the proposed approach in remotely sensed images. This will be useful in image processing for future planetary missions like Chandrayaan-2 and Mars Orbiter Mission (MOM).

\section{ACKNOWLEDGEMENT}

This work is carried out in Research and Development Center, Dharmsinh Desai University, Nadiad and with resources of PLANEX, PRL project as M.Tech Dissertation work. We sincerely thank the staff of $\mathrm{R}$ and $\mathrm{D}$ Center for providing technical support. We would also like to acknowledge the following organizations for their assistance by supplying lunar image datasets and related information: PDS-Imaging node at JPL, National Aeronautics and Space Administration (NASA) for LRO Datasets, Indian Space Science Data Center (ISSDC), ISRO for Chandrayaan1 TMC Dataset and Physics Research Laboratory (PRL).
[1] Zitova, Barbara, and Jan Flusser. "Image registration methods: a survey."Image and vision computing 21.11 (2003): 977-1000.

[2] Brown, Lisa Gottesfeld. "A survey of image registration techniques." ACM computing surveys (CSUR) 24.4 (1992): 325-376.

[3] Kirk, Randolph L., E. Howington-Kraus, and Mark Rosiek. "Recent planetary topographic mapping at the USGS, Flagstaff: Moon, Mars, Venus, and beyond." INTERNATIONAL ARCHIVES OF PHOTOGRAMMETRY AND REMOTE SENSING 33.B4/2; PART 4 (2000): 476-490.

[4] Bandeira, Lourenço, Jose Saraiva, and Pedro Pina. "Impact crater recognition on Mars based on a probability volume created by template matching."Geoscience and Remote Sensing, IEEE Transactions on 45.12 (2007): 4008-4015.

[5] Bue, Brian D., and Tomasz F. Stepinski. "Machine detection of Martian impact craters from digital topography data." Geoscience and Remote Sensing, IEEE Transactions on 45.1 (2007): 265-274.

[6] Vinogradova, Tatiana, Michael Burl, and Eric Mjolsness. "Training of a crater detection algorithm for Mars crater imagery." Aerospace Conference Proceedings, 2002. IEEE. Vol. 7. IEEE, 2002.

[7] Wetzler, Philipp Georg, et al. "Learning to detect small impact craters."Application of Computer Vision, 2005. WACV/MOTIONS'05 Volume 1. Seventh IEEE Workshops on. Vol. 1. IEEE, 2005.

[8] Magee, M., Chapman, C., Dellenback, S., Enke, B., Merline, W., Rigney, M.: Automated Identification of Martian Craters Using Image Processing. Lunar and Planetary Science XXXIV (2003) 1756

[9] Illingworth, John, and Josef Kittler. "A survey of the Hough transform." Computer vision, graphics, and image processing 44.1 (1988): 87-116.

[10] Barata, Teresa, et al. "Automatic recognition of impact craters on the surface of Mars." Image Analysis and Recognition. Springer Berlin Heidelberg, 2004. 489-496.

[11] Radhadevi, P. V., et al. "An algorithm for geometric correction of full pass TMC imagery of Chandrayaan-1." Planetary and Space Science 79 (2013): 45-51.

[12] Wu, Bo, et al. "Integration of Chang'E-1 imagery and laser altimeter data for precision lunar topographic modeling." Geoscience and Remote Sensing, IEEE Transactions on 49.12 (2011): 4889-4903.

[13] Wu, Bo, et al. "Co-registration of lunar topographic models derived from Chang'E-1, SELENE, and LRO laser altimeter data based on a novel surface matching method." Earth and Planetary Science Letters 364 (2013): 68-84.

[14] Anderson, F. Scott, et al. "Analysis of MOLA data for the Mars Exploration Rover landing sites." Journal of Geophysical Research: Planets (1991-2012)108.E12 (2003).

[15] Kirk, R. L., et al. "Ultrahigh resolution topographic mapping of Mars with MRO HiRISE stereo images: Meter-scale slopes of candidate Phoenix landing sites."Journal of Geophysical Research: Planets (19912012) 113.E3 (2008).

\section{REFERENCES}


[16] Dickson, James L., James W. Head, and Mikhail Kreslavsky. "Martian gullies in the southern midlatitudes of Mars: Evidence for climate-controlled formation of young fluvial features based upon local and global topography." Icarus 188.2 (2007): 315-323.

[17] Bhandari, Narendra. "Chandrayaan-1: science goals." Journal of earth system science 114.6 (2005): 701-709.

[18] S.S.Solanki, T.Krishna Sumanth, J.Saibaba and Geeta Varadan, "Rigorous photogrammetric processing of chandrayaan-1 terrain mapping Camera (tmc) images for lunar topographic mapping ", 42nd Lunar and Planetary Science Conference, 2011.

[19] Houghton, Martin B., Craig R. Tooley, and Richard S. Saylor Jr. "Mission design and operations considerations for NASA's Lunar Reconnaissance Orbiter." 58th International Astronautical Congress, Hyderabad, India. http://lunar. gsfc. nasa. gov/library/IAC-07-C1_7_06. pdf. 2007.

[20] Boda, Somaraju. "Feature-based image registration". Diss. National Institute of Technology Rourkela, 2009.

[21] Heath, Mike, et al. "Comparison of edge detectors: a methodology and initial study." Computer Vision and Pattern Recognition, 1996. Proceedings CVPR'96, 1996 IEEE Computer Society Conference on. IEEE, 1996.

\section{BIOGRAPHIES}
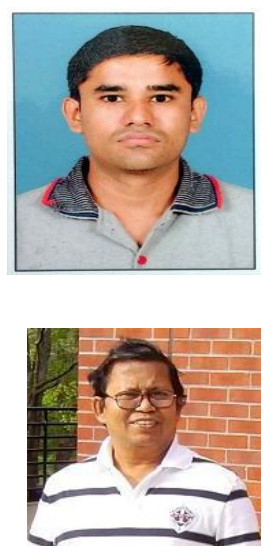

Vivek Kumar received his Bachelor degree in Computer engineering in 2013 and currently pursuing Masters in Information Technology. He has carried out dissertation work in Research and Development Center during 2014-2015 from Dharmsinh Desai University.

Himanshu S Mazumdar, Ph.D., Senior Member, IEEE is currently working as Professor, EC and Head of Research and Development Center at Dharmsinh Desai University. He has worked in important space missions in University College London, NASA's Space Shuttle and Indian Space Research Organization. 